\title{
Probing Angular Correlations in Sequential Double Ionization
}

\author{
A. Fleischer, ${ }^{1,2}$ H. J. Wörner, ${ }^{1,3}$ L. Arissian,,${ }^{1,4}$ L. R. Liu,,${ }^{1,5}$ M. Meckel, ${ }^{6}$ A. Rippert, ${ }^{6}$ R. Dörner, ${ }^{6}$ D. M. Villeneuve,${ }^{1}$ \\ P. B. Corkum, ${ }^{1}$ and A. Staudte ${ }^{1}$ \\ ${ }^{1}$ Joint Attosecond Science Laboratory, National Research Council and University of Ottawa, \\ 100 Sussex Drive, Ottawa, Ontario, Canada K1A 0R6 \\ ${ }^{2}$ Department of Physics and Solid State Institute, Technion, Haifa 32000, Israel \\ ${ }^{3}$ ETH Zürich, Wolfgang-Pauli-Strasse 10, 8093 Zürich, Switzerland \\ ${ }^{4}$ Texas A\&M University, College Station, Texas 77843, USA \\ ${ }^{5}$ University of Toronto, 40 St. George Street, Toronto, Ontario M5S 2E4, Canada \\ ${ }^{6}$ Institut für Kernphysik, Goethe Universität, Max-von-Laue Strasse 1, D-60438 Frankfurt, Germany
}

(Received 18 May 2011; published 8 September 2011)

\begin{abstract}
We study electron correlation in sequential double ionization of noble gas atoms and $\mathrm{HCl}$ in intense, femtosecond laser pulses. We measure the photoelectron angular distributions of $\mathrm{Ne}^{+}$relative to the first electron in a pump-probe experiment with $8 \mathrm{fs}, 800 \mathrm{~nm}$, circularly polarized laser pulses at a peak intensity of a few $10^{15} \mathrm{~W} / \mathrm{cm}^{2}$. Using a linear-linear pump-probe setup, we further study $\mathrm{He}, \mathrm{Ar}$, and $\mathrm{HCl}$. We find a clear angular correlation between the two ionization steps in the sequential double ionization intensity regime.
\end{abstract}

DOI: 10.1103/PhysRevLett.107.113003

PACS numbers: $32.80 . \mathrm{Rm}, 31.70 . \mathrm{Hq}, 33.80 . \mathrm{Rv}$

Electron correlation is one of the frontiers of modern science. In ultrafast laser science, electron correlation has found prominence in the dramatic enhancement of multiphoton multiple ionization [1]. The underlying mechanism, called recollision [2], forms the foundation of attosecond science [3]. Above a certain threshold intensity, the laser field-electron coupling dominates electron correlation and multiple ionization proceeds sequentially, i.e., in two independent steps $[4,5]$. This assumption has been recently called into doubt by experiments [6,7]. Specifically, in sequential ionization each successive ionization stage is assumed to lose memory of the previous one. However, increasing experimental evidence suggests that multiphoton ionization in the tunneling limit always creates a correlated hole wave packet in the parent ion [8-11], effectively storing the memory of the ionization in the valence shell of the parent ion. Recently, advances in attosecond pulse generation have enabled the real-time observation of a signature of the valence shell rearrangement following tunnel ionization [11].

Using ion momentum spectroscopy, we observe that tunnel ionization launches a periodic redistribution of the valence electrons in an atom. We measure the angular dependence of multiphoton double ionization in the socalled sequential intensity regime. We extrapolate the time-dependent angular correlation to an instantaneous double ionization. We find that, in the intensity regime usually attributed to sequential ionization, successive ionization steps are not independent.

Laser-induced tunnel ionization in linearly [12] and circularly polarized light $[13,14]$ has been shown to reveal structure and symmetry of the outermost electron orbitals and to provide insight into the tunneling process itself [15]. For circular polarization and molecules, this has been called a laser STM (scanning tunneling microscope). We apply the laser STM to image the hole left by the first tunneling electron by measuring the emission direction of the second electron. We exploit the fact that tunnel ionization projects the momentum-space orbital wave function into the continuum through a lateral low-pass (Gaussian) filter. Simultaneously, tunneling acts as an energy filter that favors removing the most weakly bound electron [16]. Since the momentum filter can be applied in any direction, it can be used to probe angular correlations between valence electrons via sequential tunnel ionization. Through the interplay of the filters, electronic correlations determine the order in which electrons can be removed and the direction in which they leave. As an example we will study $p$-shell systems, such as, e.g., noble gas atoms, and $\mathrm{HCl}$. In these systems, the $e-e$ interaction forces the degenerate orbitals to be perpendicular to each other. Hence, multiphoton multiple ionization can be expected to favor the removal of electrons from perpendicular orbitals.

The correlation between the two ionization steps can be directly observed in the interelectronic emission angle $\theta_{12}$, which characterizes the correlation between the electrons. Experimentally, $\theta_{12}$ can be retrieved if the momenta of two of the three particles - the two electrons and the ion-are measured. However, a coincidence experiment at the required intensities is, while possible, very challenging [6]. The momentum of the double ion is easily measured, however, the ion alone encodes only the center-of-mass momentum of the two electrons and hence only limited information about $\theta_{12}[6,7]$.

Figure 1(a) illustrates how the interelectronic emission angle $\theta_{12}$ can be encoded in the double ion recoil momentum by using a pulse sequence of a linearly polarized pulse followed by a circularly polarized pulse. First, in 



FIG. 1 (color online). (a) A sequence of a linearly and a circularly polarized pulse successively ionizes two electrons. The momentum of the first electron created by the linear pulse has an expectation value of zero. Hence, the interelectronic angle $\theta_{12}$ is mapped onto the recoil ion emission angle $\varphi$ in the lab frame. Inset: $\mathrm{Ne}^{2+}$ momentum distributions at $3.6 \times$ $10^{15} \mathrm{~W} / \mathrm{cm}^{2}$ per pulse. (b) Energy diagram to illustrate the pathways to the double ion.

the linearly polarized pulse, an electron tunnels along the polarization axis defining the $z$ direction-one axis for $\theta_{12}$ in laboratory space. Because of the low mean momentum transfer in linearly polarized light, the singly charged ion is left largely at rest by the first ionization step [16]. Then, the circularly polarized pulse ionizes a second electron. As before, circular polarization removes the directional constraint from the tunneling filter. Since the asymptotic electron momentum is proportional to the vector potential of the laser field at the instant of tunneling [13,14], the recoiling ion reflects the tunneling direction of the second electron. Thus, the interelectronic angle $\theta_{12}$ becomes observable in the emission angle of the double ion in the laboratory frame.

The inset in Fig. 1(a) exemplifies the resulting double ion momentum space for the case of neon subjected to two successive 8 fs pulses (linear-circular), each at a peak intensity of $3.6 \times 10^{15} \mathrm{~W} / \mathrm{cm}^{2}$. Shown is the plane of polarization, integrated over the pulse propagation direction. Radial ion momenta below 3 a.u. are exclusively due to double ionization within the linearly polarized pulse [5]. Double ionization within the circularly polarized pulse produces a nearly isotropic ion distribution. Hence, by restricting the data analysis to radial ion momenta between 3 and 8 a.u., we can resolve the variations in the interelectronic angle $\theta_{12}$ as a deviation from an isotropic distribution.

Figure 1(b) illustrates the difference of the two ionization pathways in the potential energy diagram. Generally, in noble gas atoms, tunneling from the ground state creates a vacancy in the $p_{z}\left(m_{l}=0\right)$ orbital leaving the ion in a superposition of spin-orbit states (e.g., [11,17-19]). Specifically, the two fine structure states ${ }^{2} P_{1 / 2}$ and ${ }^{2} P_{3 / 2}$ of the $\mathrm{Ne}^{+}$ion [18] drive a 42.7 fs quantum beat. For double ionization in a single few-cycle pulse, these intermediate states do not contribute to the correlation between two degenerate $p$ orbitals. The second electron is chosen among the five remaining electrons in the $p$ shell. Removing an electron from one of the doubly occupied orbitals generates $\mathrm{Ne}^{2+}$ in its ground electronic state or the first excited singlet state $\left({ }^{1} D_{2}\right)$. When the second electron is removed from the singly occupied orbital, the dication is produced in an excited electronic state $\left({ }^{1} D_{2}\right.$ or $\left.{ }^{1} S_{0}\right)$, which is disfavored by 3.2 and $6.9 \mathrm{eV}$, respectively. The electron correlation, present as the splitting of the double ion states $\left({ }^{3} P,{ }^{1} D_{2}\right.$, or $\left.{ }^{1} S_{0}\right)$, controls which electron is removed in the second step [20] and hence the angular distribution of the continuum electrons. However, in a pump-probe experiment the coherence between the ${ }^{2} P_{1 / 2}$ and ${ }^{2} P_{3 / 2}$ states leads to a periodic rearrangement of the initially aligned vacancy $[11,18]$. This quantum beat is critical to extrapolate the angular correlation $\theta_{12}$ back to $\Delta t=0 \mathrm{fs}$.

Now we briefly outline the experimental parameters. We use a $800 \mathrm{~nm}$ pulse from a Ti:sapphire oscillator-amplifier system. The pulse is compressed in a hollow core fiber [21] creating a $50 \mu \mathrm{J}, 8 \mathrm{fs}$ diffraction-limited pulse. The few-cycle pulse was split in an interferometric setup to allow independent polarization and time delay control. The resulting two pulses were recombined before the vacuum chamber by using a polarization-independent beam combiner. The laser beam was focused into a vacuum chamber where it interacted with a low density beam of gas molecules. We employ COLTRIMS to measure the threedimensional ion momentum distribution [22].

Figure 2(a) shows the measured ionization angle $\varphi$ of the $\mathrm{Ne}^{2+}$ ion in the laboratory frame as a function of the delay between a linearly and a circularly polarized $8 \mathrm{fs}$ pulse, each focused to a peak intensity of $3.6 \times$ $10^{15} \mathrm{~W} / \mathrm{cm}^{2}$. We choose our axis such that for positive delays $\Delta t>0 \mathrm{fs}$, the circular pulse trails the linear pulse. The linearly polarized pulse ionizes the neutral atom and establishes a direction of tunneling for the first ionization step. The circularly polarized pulse then probes the tunneling anisotropy in the $\mathrm{Ne}^{+}$ion. Hence, in this configuration the double ion recoil angle $\varphi$ corresponds to the interelectronic angle $\theta_{12}$.

For $\Delta t>0 \mathrm{fs}$, the correlation manifests itself as a periodic change of the anisotropy in $\varphi$ and hence $\theta_{12}$. In the temporal overlap region $\Delta t=0 \mathrm{fs}$, the effective laser field is changing rapidly in polarization and amplitude. When the electric fields of the two pulses interfere constructively, ionization along one axis $\left(0^{\circ}\right.$ or $\left.180^{\circ}\right)$ is strongly preferred, independent of any angular correlation between the two ionization steps. If the overlapping pulses shift by half a wavelength, the two field components cancel partially. Consequently, at 


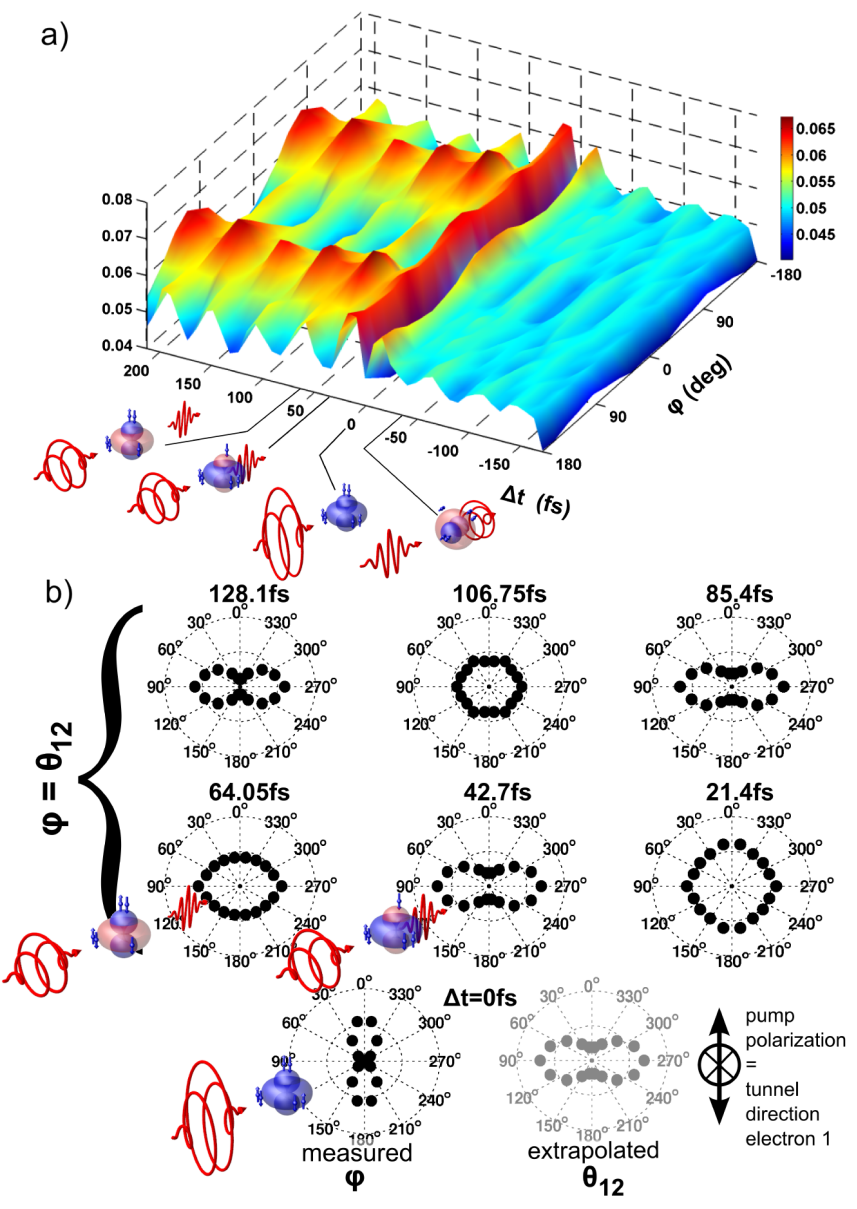

FIG. 2 (color online). (a) Yield of $\mathrm{Ne}^{2+}$ as a function of the lab frame angle of ionization $\varphi$ and of the delay $\Delta t$ between two 8 fs pulses. For positive delays the linearly polarized pulse precedes the circular pulse. For $\Delta t>0$ fs the $\mathrm{Ne}^{2+}$ emission angle $\varphi$ corresponds to the interelectronic angle $\theta_{12}$. Data are normalized (see the text). (b) Representation of $d N / d \theta_{12}$ in polar coordinates for positive pump-probe delays. Global radial minimum is $\min \left[d N / d \theta_{12}(\Delta t=0 \mathrm{fs})\right]$. Measured distribution at $\Delta t=0 \mathrm{fs}$ (scaled $\times 3$ for better comparison) reflects the polarization ellipse of the overlapping pulses. Extrapolation from $\Delta t>0 \mathrm{fs}$ reveals that the preferred $\theta_{12}$ at $\Delta t=0$ fs is $90^{\circ}$ and $270^{\circ}$.

$\Delta t=0 \mathrm{fs}$, the total ionization yield is greatly suppressed and $\varphi$ is dominated by the anisotropy in the polarization ellipse. At negative delays $\Delta t<0 \mathrm{fs}$, the circular pulse precedes the linear pulse. In this configuration the laser STM is being applied to the neutral atom. Therefore, apart from a remaining ellipticity in the circular pulse, an isotropic distribution of $\varphi$ is to be expected. Hence, we normalized the data with an angular distribution averaged over the negative delays only in order to remove any residual ellipticity in the circularly polarized pulse.

We now examine the correlation in the interelectronic angle $\theta_{12}$. In Fig. 2(b), $\varphi$ is presented in polar coordinates for positive pump-probe delays. At multiple integers of the quantum beat period $\left(t_{0}=42.7 \mathrm{fs}\right)$, a strong correlation in the tunneling probability for perpendicular emission $\left(\left\langle\theta_{12}\right\rangle=90^{\circ}\right)$ is observed. This correlation is lost at half integer multiples $(n+1 / 2, n=0,1,2)$ of $t_{0}$ resulting in a largely isotropic emission of the second electron with respect to the first one. At $\Delta t=0$ fs the double ion emission angle $\varphi$ peaks around $0^{\circ}$ and $180^{\circ}$, directly corresponding to the polarization ellipse of the combined circularly and linearly polarized pulses. Extrapolating the time-varying distribution back to $\Delta t=0 \mathrm{fs}$ shows that successive tunnel ionization proceeds preferentially along perpendicular directions. Our laser STM directly images the time-dependent electron density in the ionic valence shell initiated by tunneling [23]. In a multiphoton manifestation of Hund's rule [24], the second ionization is suppressed for parallel polarizations of the short pulses: The directional tunneling filter has great difficulty removing the remaining electron from the $p_{z}$ orbital.

We now generalize and simplify the experiment once more. Instead of measuring the ion recoil momentum, which becomes increasingly difficult for heavy atoms or molecules, it is sufficient to measure ionization probability by using two linearly polarized pulses. With independently controlled linearly polarized pulses, we probe the directional probability of the second ionization of the valence shell at any time delay observing the alignment of the vacancy. In addition to observing the hole wave packet evolve, we can again extrapolate back to $\Delta t=0 \mathrm{fs}$ to determine the initial correlation.

Figure 3(a) shows the time-dependent ratio of double to single ion yield from two colinearly polarized pulses for three noble gases. The ratio removes some of the noise resulting from energy fluctuations in the laser beam. At delay $\Delta t=0 \mathrm{fs}$, the two pulses overlap, yielding a strong autocorrelation peak in the double ion yield for all three species, due to the strong nonlinear nature of tunnel ionization. While the relative double ion yield shows no structure in $\mathrm{He}$, it is strongly modulated for $\mathrm{Ne}$ and $\mathrm{Ar}$. By extending one arm of the interferometer, we observe that the modulations sustain over thousands of periods (inset). The modulations are substantial and modulate the absolute yield of the double ion. In Fig. 3(b), the absolute yield (black solid line) of $\mathrm{Ne}^{2+}$ at an intensity of $1.7 \times 10^{15} \mathrm{~W} / \mathrm{cm}^{2}$ is compared to an analytically derived ionization yield (black, red, and blue lines) according to a simple model published elsewhere [20].

We now demonstrate how the angular correlation can be extracted from the double ion yields by using linear pulses only. In Fig. 3(c), we compare the cases where pump and probe pulses are perpendicular (top line) and parallel (bottom line). The time-dependent yields are $90^{\circ}$ out of phase. Extrapolating each curve back to $\Delta t=0 \mathrm{fs}$, we find that the instantaneous emission of the two electrons is suppressed with parallel polarizations in the pump and probe. Emission occurs preferentially in perpendicular directions - a natural consequence of the multielectron orbital structure of several filled $p$ orbitals. 

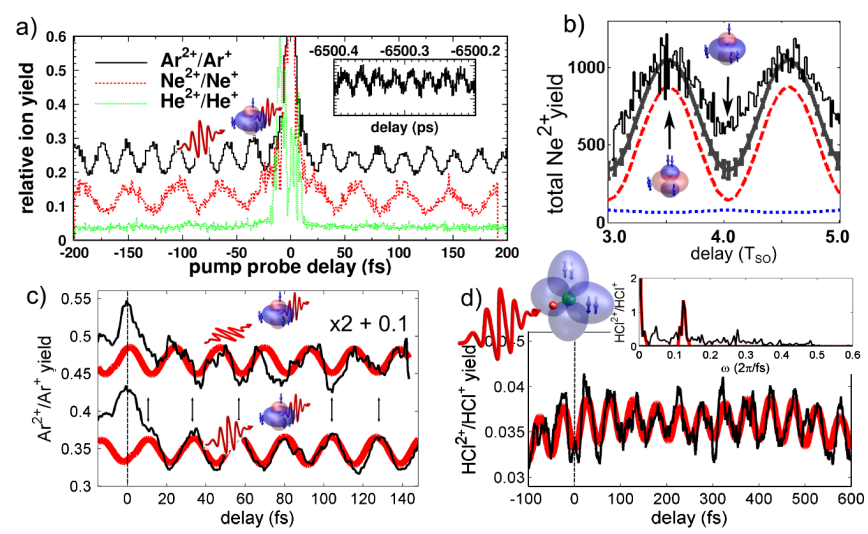

FIG. 3 (color online). Time-dependent double ion yields for linearly polarized 8 fs pulses. (a) Relative yields for parallel polarizations for helium $\left(3.4 \times 10^{15} \mathrm{~W} / \mathrm{cm}^{2}\right)$, neon $(2.5 \times$ $\left.10^{15} \mathrm{~W} / \mathrm{cm}^{2}\right)$, and $\operatorname{argon}\left(1.2 \times 10^{15} \mathrm{~W} / \mathrm{cm}^{2}\right)$. Inset: $\mathrm{Ar}^{2+} / \mathrm{Ar}^{+}$, long delays. (b) Absolute $\mathrm{Ne}^{2+}$ yield at $1.7 \pm 0.7 \times$ $10^{15} \mathrm{~W} / \mathrm{cm}^{2}$ compared to calculation [20]: yield from the $p_{z}$ $\left(m_{l}=0\right)$ orbital (dashed, red line), yield from the $p_{x y}\left(m_{l}= \pm 1\right)$ orbital (dotted, blue line), and total yield (solid, black line). (c) $\mathrm{Ar}^{2+} / \mathrm{Ar}^{+}$ratios for perpendicular (top) and parallel (bottom) relative polarizations $\left(1.9 \times 10^{15} \mathrm{~W} / \mathrm{cm}^{2}\right)$. Superimposed are inverse Fourier transforms of the strongest peak in the frequency spectrum (red line). Note that the upper trace has been scaled and shifted for presentation purposes. (d) $\mathrm{HCl}^{2+} / \mathrm{HCl}^{+}$yield as a function of the pulse delay for parallel polarizations $\left(4.8 \times 10^{15} \mathrm{~W} / \mathrm{cm}^{2}\right)$. Inverse Fourier transform (red line) filtered around the dominant frequency at $0.13 \mathrm{rad} / \mathrm{fs}$ is superimposed (inset).

The time-dependent $\mathrm{HCl}^{2+}$ yield in Fig. 3(d) confirms this conclusion. The quantum beat $\left(t_{0}=52 \mathrm{fs}\right)$ due to the two fine structure states is visible in the frequency spectrum as a peak near $0.13 \mathrm{rad} / \mathrm{fs}$ [25]. Thus, sequential tunnel ionization of $\mathrm{HCl}$ measures the correlation dynamics and therefore the correlation in even a randomly aligned molecular sample. The $\mathrm{HCl}^{2+} / \mathrm{HCl}^{+}$ratio exhibits only a weak autocorrelation peak contrasting the atomic case. Around the temporal overlap the dissociation of the molecular ion is enhanced, depleting the $\mathrm{HCl}^{2+}$ signal. Tunnel ionization coherently excites several vibrational states in the molecular ion [26], which further modulate the timedependent ionization rate and are responsible for the high-frequency components in the pump-probe trace. Furthermore, a much faster electronic wave packet of about $1 \mathrm{fs}$ period can be created by ionization from highest occupied molecular orbital HOMO-1 of $\mathrm{HCl}$ in the first step. For molecules that are aligned perpendicular to the laser field, the contribution of HOMO-1 can reach $10 \%$ [13]. However, the employed pulse durations used in the current experiment would likely destroy the coherence between HOMO and HOMO-1 [18], a limitation that can be overcome by scanning the wavelength [20].

In conclusion, we have demonstrated that tunneling can image atomic valence orbitals and their dynamics, just as it images static molecular orbitals [27]. In our case, the orbital was prepared by tunneling using a pump laser pulse. Tunneling is only one way to create electronic dynamics in an atom or molecule. Any temporally confined ionization projects the correlations that exist in the neutral ground state onto the electronic states of the ion. Imaging its electronic structure and scanning its time evolution amounts to characterizing the correlations of the initial wave function. Since our pulses can be perfectly synchronized with attosecond extreme ultraviolet pulses, an attosecond pulse can launch multielectron dynamics, which is then studied by tunneling one or more electrons from the system.

This project was supported by AFOSR, DAAD, ESROP SRF, the German National Academic Foundation, the Koselleck-Project of the DFG, NSERC, U.S. Army MURI, and Yad Hanadiv. We thank S. Patchkovskii for enlightening discussions. We are indebted to T. Jahnke, Cronologic, B. Avery, D. Crane, and A. Naumov for technical support. We gratefully acknowledge help from $\mathrm{R}$. Lausten, C. Bisgaard, F. Turner, N. Kajumba, C. Trallero, M. Gertsvolf, and E. Frumker.

Note added in proof.-Recently, ab initio simulations of interrogating orbital structure with a circularly polarized laser pulse have become available [28].

[1] B. Walker et al., Phys. Rev. Lett. 73, 1227 (1994).

[2] P. B. Corkum, Phys. Rev. Lett. 71, 1994 (1993).

[3] F. Krausz and M. Yu. Ivanov, Rev. Mod. Phys. 81, 163 (2009).

[4] Th. Weber et al., Nature (London) 405, 658 (2000).

[5] A. Rudenko et al., Phys. Rev. Lett. 93, 253001 (2004).

[6] A. N. Pfeiffer et al., Nature Phys. 7, 428 (2011).

[7] C. M. Maharjan et al., Phys. Rev. A 72, 041403(R) (2005).

[8] B. K. McFarland et al., Science 322, 1232 (2008).

[9] W. Li et al., Science 322, 1207 (2008).

[10] O. Smirnova et al., Nature (London) 460, 972 (2009).

[11] E. Goulielmakis et al., Nature (London) 466, 739 (2010).

[12] A. S. Alnaser et al., Phys. Rev. Lett. 93, 113003 (2004).

[13] H. Akagi et al., Science 325, 1364 (2009).

[14] A. Staudte et al., Phys. Rev. Lett. 102, 033004 (2009).

[15] P. Eckle et al., Science 322, 1525 (2008).

[16] L. V. Keldysh, Sov. Phys. JETP 20, 1307 (1965).

[17] L. Young et al., Phys. Rev. Lett. 97, 083601 (2006).

[18] N. Rohringer and R. Santra, Phys. Rev. A 79, 053402 (2009).

[19] D. Shafir et al., Nature Phys. 5, 412 (2009).

[20] H. J. Wörner and P. B. Corkum, J. Phys. B 44, 041001 (2011).

[21] M. Nisoli et al., Opt. Lett. 22, 522 (1997).

[22] J. Ullrich et al., Rep. Prog. Phys. 66, 1463 (2003).

[23] Z. H. Loh et al., Phys. Rev. Lett. 98, 143601 (2007).

[24] R. J. Boyd, Nature (London) 310, 480 (1984).

[25] R. F. Fink et al., Phys. Rev. A 65, 034705 (2002).

[26] Th. Ergler et al., Phys. Rev. Lett. 97, 193001 (2006).

[27] M. Meckel et al., Science 320, 1478 (2008).

[28] M. Abu-Samha and L. B. Madsen, Phys. Rev. A 84, 023411 (2011). 